\title{
A Distributed and Dynamic Data Gathering Protocol for Sensor Networks*
}

\author{
Hüseyin Özgür Tan, \\ Bilkent University \\ Bilkent, Ankara, Turkey \\ hozgur@cs.bilkent.edu.tr
}

\author{
İbrahim Körpeoğlu \\ Bilkent University \\ Bilkent, Ankara, Turkey \\ korpe@cs.bilkent.edu.tr
}

\author{
Ivan Stojmenović \\ SITE, University of Ottawa \\ Ottawa, Ontario, Canada \\ ivan@site.uottawa.ca
}

\begin{abstract}
In this paper we propose a distributed, self organizing, robust and energy efficient data gathering algorithm for sensor networks operating in environments where all the sensor nodes are not in direct communication range of each other and data aggregation is used while routing. Proposed algorithm is based on local minimum spanning tree (LMST) structure, which nodes can construct from the position of their 1-hop neighbors. Reporting tree is constructed from the sink by allowing only edges of LMST to join the tree, plus possibly some direct links to the sink. Each node selects as parent the LMST neighbor so that the total energy cost of route to the sink is minimal. We also describe route maintenance protocols to respond to predicted sensor failures and addition of new sensors. Our simulation results show that our algorithm prolongs the network lifetime significantly compared to some alternative schemes.
\end{abstract}

\section{Introduction}

The design of wireless sensor networks depends on the application requirements. Environmental monitoring is an application where a region can be sensed by numerous sensor nodes and the sensed data are gathered at a base station (a sink) where further processing can be performed. The sensor nodes for such applications are usually designed to work in conditions where it may not be possible to recharge or replace the batteries of the nodes. This means that energy is a very precious resource for sensor nodes, and communication overhead is to be minimized. Processing power is also limited. These constraints make the design of data communication protocols a challenging task.

A common scenario of sensor networks involves deployment of hundreds or thousands of low-cost, low-power sensor nodes to a region from where information will be col-

\footnotetext{
*This work is supported by The Scientific and Technological Research Council of Turkey with Project EEEAG 104E028, NSERC Strategic Project STPGP 336406 and NSERC Discovery grant.
}

lected periodically. Hence sensor nodes will periodically sense their nearby environment and send the information to a sink which is not energy or computing power limited. At the sink the collected information can be further processed for end-user queries. In order to reduce the communication overhead and energy consumption of sensors, received data are combined to reduce message size. A simple way of doing that is aggregating (sum, average, min, max, count) the data. A different and elegant way is data fusion which can be defined as producing a more accurate signal by combining several unreliable data measurements.

An important problem here is finding an energy efficient routing scheme for gathering of all data at the base station periodically so that the lifetime of the network is prolonged as much a possible. The lifetime can be expressed in terms of rounds where a round is the time period between two sensing activities of sensor nodes.

There are several requirements for a routing scheme to be designed for this kind of scenarios. First, the algorithm should be distributed since it is extremely energy consuming to calculate the optimum paths in a dynamic network and inform others about the computed paths in a centralized manner. The algorithm must also be scalable. The message and time complexity of computing the routing paths must scale well with increasing number of nodes. Another desirable property for a routing scheme is robustness, which means that the routing scheme should be resilient to node and link failures. The scheme should also support new node additions to the network, since not all nodes fail at the same time, and some nodes may need to be replaced. In other words, the routing scheme should be self-healing. The final and possibly the most important requirement for a routing scheme for wireless sensor networks is being energy efficient.

In this work we propose an LMST based routing scheme which satisfies the requirements stated above. Our scheme is distributed and requires each sensor node to compute a local minimum spanning tree (MST) spanning just the local environment, i.e. the node and its neighbors. The local MSTs of individual sensors are then combined to make up 
a structure called LMST [8] that is spanning all the nodes in the network. LMST does not have to be tree; it can be a graph. After obtaining the LMST structure spanning all the nodes in the network, we run a shortest weighted path tree algorithm from the sink, allowing only edges of LMST and direct links to sink to join it. We route the packets on the edges of this shortest weighted path tree.

Our scheme can also handle new node arrivals and departures of existing nodes (due to mobility or due to energy exhaustion). Hence it is adaptive. The routing path is maintained when those dynamic conditions occur.

The rest of the paper is organized as follows. In Section 2, we briefly discuss the related work. In Section 3, we define our system model and describe the problem we solve. In Section 4 we provide our solution. In section 5 we present our simulation results. And finally in Section 6, we conclude the paper and discuss about some future directions of research.

\section{Related Work}

Singh et al [13] proposed to apply Dijsktra's shortest weighted path algorithms, using hop count, transmission power, and reluctance to forward as metrics. Stojmenovic and Lin [14] proposed localized power and reluctance aware routing protocols, where nodes select best neighbor to forward based only on the knowledge of 1-hop neighbors and destination. Chang and Tassiulas [4, 3] apply distributed Bellman-Ford shortest weighted path algorithm, using several combinations of transmission power and reluctance (inversely proportional to the remaining energy) as metrics. The goal is to maximize the system lifetime. Their algorithm is called MREP (minimum residual energy route). Another alternative approach is MBCR (minimum battery cost route) [16] in which only the remaining energy is considered for route computation. In this scheme the optimal path is the one with minimum total reluctance.

Rodoplu and Meng [12] propose the enclosure graph. An edge $A B$ is in the enclosure graph if and only if the direct transmission between $A$ and $B$ consumes less energy than the total energy of all links of any path between them. They propose also to charge constant energy $c$ for transmission at distance 0 .

Heinzelman et al. [5] propose a 2-level hierarchy. Sensors randomly decide whether or not to become clusterheads. If not, they join nearest clusterhead and transmit measured data to it. Clusterheads aggregate collected data and transmit directly to the sink. A number of subsequent protocols proposed improvements to this LEACH protocol. Lindsey et al. [9] proposed PEGASIS protocol. Sensors are organized into a chain by a centralized algorithm. They transmit to each other along the chain, aggregate received data, and last sensor in the chain transmits to the sink.
Bhardwaj et al. [1] derived upper bounds on the lifetime of sensor networks. For the case where all sensor nodes can directly communicate with each other, Kalpakis et al. [7] propose a linear programming solution to maximize the lifetime. The solution provides near optimal results. Their approach, however, has high computational cost and must be applied in a central location.

In [15] Tan and Korpeoglu showed that their protocol PEDAP, which routes the packets on the edges of the minimum spanning tree (MST), improves the system lifetime dramatically. In that work the authors set the link costs as the energy consumption of sending data on the corresponding link. The main idea behind the success of the scheme is to minimize the total energy consumption in a round, while balancing the load equally among the sensor nodes. As we will show in Section 3, the energy load of a node is directly related with its degree. For a single round, the minimum energy consumption can be achieved by an MST, and fortunately the MST has a maximum node degree of 5 which can be seen under very rare conditions. Thus, PEDAP outperforms its alternatives. Moreover the authors propose a power aware version of their protocol PEDAP, which they call PEDAP-PA, by changing the link costs so that the remaining energy level of the sending node is also considered. It is shown that PEDAP-PA protocol increases the lifetime of the first node considerably. As a result of this work, one can conclude that using an MST structure is suitable for environments where all the nodes have data to send and the data can be aggregated or fused in the relay nodes. The drawback is the centralized nature of MST, and the lack of quick response to node failures.

There are also protocols based on minimizing hop count and delay instead of energy consumption. The classical routing algorithms such as AODV [11] or Directed Diffusion [6] can be considered for this case. They are based on initial blind flooding of the network, where each sensors retransmits route discovery message exactly once.

Li et al. [8] propose a neighborhood structure called local MST (LMST) as an alternative to enclosure graph. LMST is computed as follows. First, each sensor node determines its one-hop neighbors and computes an MST for that set of nodes. In order to compute the local MST, a node uses the distance between nodes as the weight of edges. After computing the MST of the neighbors, each node $i$ selects the edges $(i, j)$ where node $j$ is a direct neighbor of node $i$ in the local MST. The resulting structure is a directed graph. The structure can be converted to an undirected one in two ways [8]. First way is to include edge $(i, j)$ only when both nodes $i$ and node $j$ include that edge (LMST ${ }^{-}$). The second way is to include that edge when either node $i$ or node $j$ include it $\left(\mathrm{LMST}^{+}\right)$.

There are some desirable properties of the LMST structure which make using the structure in the context of sensor 
networks advantageous. It is proved that LMST structure is connected when the sensor network is connected. It is also proved that the MST of a graph is a sub-graph of its LMST structure [10]. The maximum degree of a node is bounded by 6 as it is in MST. This is a desirable property since the load of a node is directly related to the degree of the node as it is shown in Section 3. Another important advantage of using LMST is that it can be constructed very efficiently in a localized manner. Node deletions and additions do not globally change the structure. Only local changes in the structure is required and they can be efficiently computed when a node fails or when a new node is introduced to the network. In [8] the authors compare their LMST structure with the enclosure graph and find out that the enclosure graph performs better in terms of energy consumption. However, the comparison did not consider the effect of data aggregation.

In this paper we propose a routing solution for wireless sensor networks where the data aggregation is used inside the network in order to save energy. Our scheme provides a distributed mechanism to build up routing paths that are also power efficient. In other words, we propose a distributed routing solution that improves the lifetime of the network. In this respect, we are providing a complete data gathering solution including discovery and establishment of efficient routing paths and maintenance of them.

\section{Model and Problem Statement}

There are various sensor network application scenarios, and depending on the scenario the requirements for a routing solution is different. The following are our assumptions about the features of sensor networks and application scenarios we consider in this paper.

The sensor nodes are homogeneous and energy constrained. Sensor nodes and base station are stationary and located randomly. Every node knows the geographic location of itself. Every node senses periodically its nearby environment and has data to send to the base station in each round. The sensor nodes have a maximum transmission range which is denoted with $R$. So all of the sensors are not in direct communication with each other, and therefore centralized approaches can not be applied. Data fusion or aggregation is used to reduce the data volume. We also assume that the sensing period (the duration of a round) is much larger than the time required for transmitting all the information from all nodes to the base station.

\subsection{Energy Consumption Model}

There are different models proposed for modeling energy consumption in a sensor network and in sensor nodes. Here we use the first-order radio model described in [5] as the model for energy consumption during data transmission between two nodes. In this model, the energy consumed to transmit a $k$ bit packet to a distance $d$ (denoted as $E_{t x}$ ) and the energy consumed to receive a $k$ bit packet (denoted as $\left.E_{r x}\right)$ are given as follows:

$$
\begin{aligned}
E_{t x}(k, d) & =E_{\text {elec }} \times k+E_{\text {amp }} \times k \times d^{2} \\
E_{r x}(k) & =E_{\text {elec }} \times k .
\end{aligned}
$$

In this model, $E_{\text {elec }}$ the energy consumption of the transmit and receiver electronics is $50 \mathrm{~nJ} /$ bit and $E_{a m p}$ the energy requirement for transmit amplifier is $100 \mathrm{pJ} / \mathrm{bit} / \mathrm{m}^{2}$.

According to this model, if we express the total energy cost of transmitting a $k$ bit packet from a node $i$ to a node $j$ as $C(i, j)$, and similarly the total cost of transmitting a $k$ bit packet from a node $i$ to the base station as $C_{b}(i)$, then $C(i, j)$ and $C_{b}(i)$ are given as follows:

$$
\begin{aligned}
C(i, j) & =2 \times E_{\text {elec }} \times k+E_{\text {amp }} \times k \times d_{i j}^{2} \\
C_{b}(i) & =E_{\text {elec }} \times k+E_{\text {amp }} \times k \times d_{i b}^{2} .
\end{aligned}
$$

The costs of transmission of one packet to another node and to the base station are different since the base station has no energy constraints and because of that the receive cost of the base station is ignored. [15] showed that choosing the link costs as above provides advantages in increasing the number of nodes that directly communicate to the sink since $E_{\text {elec }}$ is quite large compared to $E_{a m p}$.

Assuming that we are routing the data packets on a tree rooted at the sink (hence a sensor node receives data from several children and transmits to a single parent after aggregation), the total energy consumed at a node $i(E(i))$ for receiving a $k$ bit packet from its children and for sending the packet to its parent is given as:

$$
E(i)=\operatorname{degree}(i) \times E_{\text {elec }} \times k+E_{\text {amp }} \times k \times d^{2}
$$

As shown in the equation above, it is also important to minimize the average degree for a node, while minimizing the distance that the node will transmit in order to minimize the energy load of the node.

\subsection{Problem Statement}

We model the reachability in a sensor network using a visibility graph $G=(V, E)$, where $V$ is the set of sensor nodes and the base station, and $E$ is the set of edges $(i, j)$, where the distance between node $i$ and $j$ is smaller than the maximum transmission distance $R$. Figure 1 shows an example network with 100 nodes and a maximum transmission range of $20 \mathrm{~m}$. In the application scenario we consider for this network model, sensor nodes periodically sense the environment and generate data in each round of communication. We assume that the time period of sensing the environment is much bigger than the time required for transmitting all the data to the base station. Given a routing plan, 


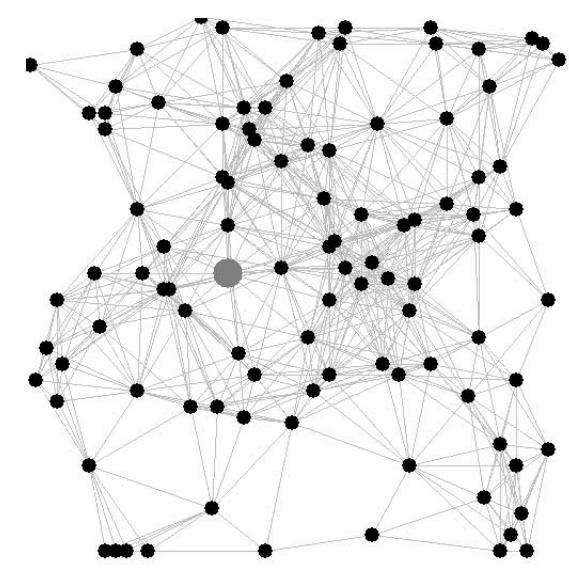

Figure 1. A sample network (100 nodes)

each sensor node receives the data from its neighbors, aggregates or fuses them into one single packet, and sends the packet to the node on its way to the base station.

The problem is to find an energy efficient routing plan which maximizes the network lifetime under the system model given above. The routing plan determines, for each round, the incoming and outgoing neighbors for data forwarding and aggregation. In other words, for each round, a tree spanning all the nodes must be found. The routing scheme should also be sensitive to node failures and should support new node arrivals.

In the context of sensor networks, the network lifetime can be defined in various ways. One of the definitions can be the time elapsed in terms of rounds until the first node depletes all of its energy. Another alternative definition can be the time elapsed until the network is partitioned so that some of the sensor nodes cannot transmit their data to the base station. It is desirable that a routing scheme considers all lifetime definitions and provide reasonably good results for all definitions. In our work we provide results related to the timings of node failures until the network is partitioned.

\section{Proposed Algorithm}

\subsection{Our Approach}

As mentioned in Section 2, the network lifetime can be considerably extended by gathering packets on the MST structure. However, computing MST requires a global knowledge about the network and has a high cost when it is attempted to be computed in a distributed manner. In this work we propose a data gathering scheme that combines the energy efficient features of the MST with the shortest weighted path tree (SPT) computed by flooding from the sink. Our aim is to efficiently compute the routing paths while the resulting paths give a superior network lifetime. Our proposed scheme is based on local minimum spanning tree (LMST) [8] structure that can be efficiently computed in localized manner. The basic idea of our algorithm is as follows. We first compute the LMST structure by using the link costs given in equations 3 and 4 and then route the packets on the tree yielded by forming a shortest weighted path tree rooted at the sink using only the edges of the LMST structure. We construct the routing paths from all nodes to the sink via a flooding based broadcast process starting from the sink and going through all the sensor nodes. This is required because a sensor node far away from the sink does not know to which node it must send the data in order to reach the sink. Since the broadcasting is done only on the edges of the LMST, we expect that the resulting tree will be a good approximation of the MST.

\subsection{Algorithm Details}

In our proposed routing scheme, at any time each sensor node knows its all one-hop neighbors and their locations, the LMST neighbors, the parent node that it will send the data to in order to reach the sink, and the child nodes that it will receive the data from before it sends the fused or aggregated packet to its parent node. Our solution consists of three parts: Route Computation, Data Gathering and Route Maintenance.

\subsubsection{Route Computation}

The main target in this phase is to setup the routes, which means determining the child and parent nodes for each node. At the end of this phase the data aggregation tree rooted at base station will be constructed.

Initially, the nodes and the sink are not aware on the environment. In the setup phase, all nodes and the sink broadcast a HELLO message, which includes their location, using their maximum allowed transmit power. After receiving HELLO messages, all nodes are informed about their onehop neighbors and their locations. Each node can then locally compute the MST structure of its one hop neighbors and initialize its LMST neighbor lists as the direct neighbors in the computed MST. After finding its LMST neighbors, node can join the distributed route computation process in order to find its parent and the child nodes on the aggregation tree.

The route computation is done via a broadcasting process which starts at the sink. The sink initiates a ROUTE-DISCOVERY packet in order to find and setup the routes from all sensor nodes toward itself. When a sensor node receives a ROUTE-DISCOVERY packet, it broadcasts the packet to all its LMST neighbors. The shortest path tree rooted at the sink is computed over the LMST structure. Each ROUTE-DISCOVERY packet has three fields: a 
sequence ID, which is increased when a new packet is initiated, a distance field which shows the cost of reaching the sink, and a neighbor list field which is the list of the LMST neighbors of the sending node. The cost of an edge is the energy cost which is computed by using the energy model given in Section 3. Sequence IDs are decided by sinks when they initiate route constructions. The packet also includes the sink's LMST neighbors.

Upon receiving a new ROUTE-DISCOVERY packet, the sensor node compares the neighbor list in the packet with its own LMST neighbor list. If $\mathrm{LMST}^{-}$is used, the node ignores (for route computation) the packet if the sender is not included in its LMST list or it is not included in the sender's LMST list. If $\mathrm{LMST}^{+}$is applied, the packet is not ignored if one of the lists includes other node. If a node does not ignore the packet, it checks its routing table and performs updates on the table if necessary. An update is necessary if a new route with a smaller cost is detected or a new ROUTE-DISCOVERY packet with a larger sequence ID is received. If a sensor node chooses to update its table, it broadcasts a ROUTE-DISCOVERY packet to all its LMST neighbors informing the new distance to the sink. It also includes its own LMST neighbor list in the packet instead of the one received. Additionally, if the node chooses to update its table and the parent node is changed, the node informs the old and new parent node about the changes so that they can update their children lists. At the end of route discovery, the routes and data aggregation tree are setup and stabilized. This means that each node knows from which neighbors it will receive data and to which node it will send the received data after aggregation.

There are several advantages of this algorithm with respect to the energy consumption along computed routes. By using only the edges of LMST structure, the overhead of control packets is decreased compared to the centralized computation of the shortest weighted path (SPT) of the initial graph at the sink, which requires that the positions of all the sensors are first gathered at the sink. Since only the LMST edges are used in route computation phase, the energy used in this broadcast operation is just enough to reach the most distant LMST neighbor instead of the energy required to send to the maximum transmission range $R$ (this saving is only applicable if $\mathrm{LMST}^{-}$is chosen).

\subsubsection{Data Gathering}

After the parent and children nodes for an individual sensor node are determined, the node can join the data gathering process. In data gathering phase each sensor node periodically senses its nearby environment and generates the data to be sent to the base station. However, before sending it directly to the parent node, it will wait all the data from its child nodes and aggregate the data coming from the child nodes together with its own data, and then send the aggregated data to the parent node. Thus, at the beginning of data gathering step, only leaf nodes can transmit their data to their corresponding parent nodes. At each step the data is gathered upwards in the tree and reaches the sink after $h$ steps, where $h$ is the height of the aggregation tree. The reason for waiting to receive data from child nodes is to use the advantage of the aggregation. In this way each sensor only transmits once in a round, and as a result saves its energy.

\subsubsection{Route Maintenance}

After determining the routes only two events can cause a change in the routing plan: node failure and node addition. We will discuss them separately.

Node failures can be due to various reasons. However the most critical reason of failures is depletion of energy in the node. Previous approaches (e.g. [5, 15, 9]) did not discuss the node failure problem. However, in these approaches a node failure in communication phase will cause a routing problem in which the descendants of the failed node cannot send their data until next setup phase. In order to prevent this, the failures must be handled as soon as possible. In our solution we handle the case where failure is due to energy depletion. However the idea behind the solution can be applied to other failure causes.

Failure of a node due to energy depletion can be handled gracefully, since the node can predict that it will die due to energy limitation. In our solution, when a node's energy reduces below a threshold value, which can be set to a very small value, the node broadcasts a BYE message using maximum allowed transmit power. All nodes receiving the BYE message will immediately update the LMST structure locally. This message is not required to be retransmitted since the node failures do not affect the structure globally. However, in this case the nodes that cannot reach the sink because of the energy depletion of their ancestor must find a new cost-efficient path to send their packets. In our solution, this is handled in a localized manner as follows. The child nodes of the failed node that receive the BYE message reset their routing tables and enter the parent-discovery phase by broadcasting a special message PARENT-DISCOVERY to its LMST neighbors. According to the receiver of that special message, if the sender is its own parent on the way to the base station, the receiver also reset its routing table and broadcasts a PARENT-DISCOVERY packet to its LMST neighbors. In this way, all the nodes that should enter the parent discovery phase will be reached. If the PARENT-DISCOVERY packet is received by a node that considers the sender of the packet as its LMST neighbor and has a valid parent and a valid cost function, the receiver constructs a new ROUTE-DISCOVERY packet as mentioned above and broadcasts it to the sender. This 


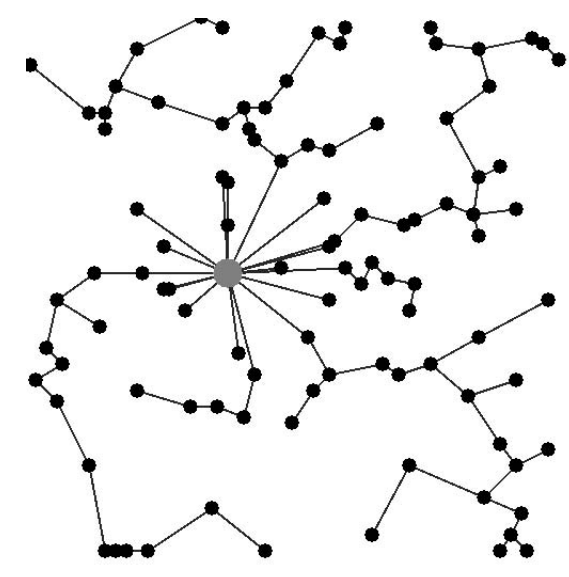

Figure 2. MST based routing scheme.

ROUTE-DISCOVERY packet is handled as mentioned in Section 4.2.1. It is worth to mention that the sequence ID in this new packet is not incremented, therefore the update of the routing table takes place only when the newly received cost is smaller. After the route discovery phase converges, the new routes are set up and data gathering can continue.

Consider now the case of node additions. When a new node is deployed, it broadcasts a HELLO message. Its neighbors update their LMST structure upon receiving this HELLO message, and also inform the new node about their existence and locations by replying a HELLO message so that the newly deployed node can also determine its LMST neighbors. Nodes that update their LMST structure send back a ROUTE-DISCOVERY packet including their costs to the newly deployed node. The new node selects the most efficient node as its parent, and broadcasts this information by a new ROUTE-DISCOVERY packet. Since the sequence ID is again not incremented, the new packet is broadcasted throughout the network only when using the new node is advantageous.

If node failures and node additions occur frequently, within a small time period, a recomputation of whole aggregation tree will be more efficient, instead of handling each event separately. In order to realize such a solution only action that a node must take is to piggyback a REROUTE bit to the data packet sent to its parent if it has still one parent. Upon receiving a data packet with a REROUTE bit piggybacked to it, the base station constructs a new ROUTE-DISCOVERY packet and initiates a new route computation phase.

\section{Simulation Results}

In order to evaluate the performance of our algorithm we implemented six different routing schemes: MST, LMST,

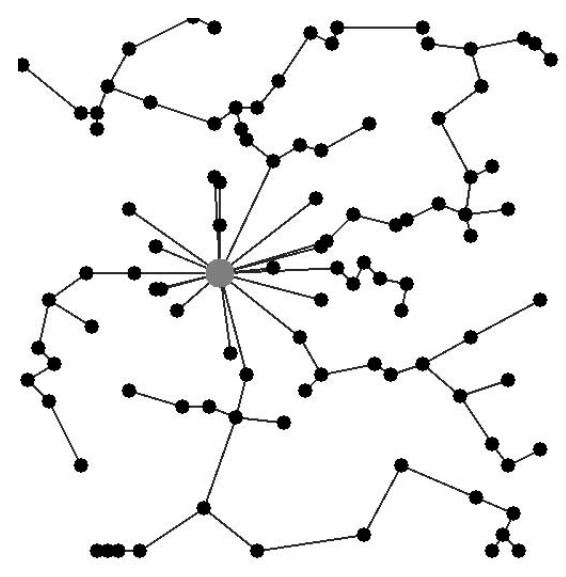

Figure 3. LMST based routing scheme.

minimum energy route obtained by constructing shortest weighted path tree with edge weight accounting for transmission and reception powers (SPT), minimum hop route, minimum battery cost routing (MBCR) [16], and maximum residual energy route (MREP) [3]. In our simulations we choosed $\mathrm{LMST}^{-}$since it approximates MST better.

The simulations were done in Java. For our simulations we generated networks of diameter $100 \mathrm{~m}$. We repeated the experiments for sensor networks having 50 and 100 nodes. We fixed the maximum transmission range of the sensors to $20 \mathrm{~m}$. The initial energies of the nodes were given as $1 \mathrm{~J}$. For power aware algorithms (MBCR and MREP) we recomputed the routing paths every 100 rounds. For transmission costs we used the parameters of the first order radio model given in [5]. In all of the routing schemes data aggregation is used at every step for a fair comparison. Also for all methods the setup and maintenance costs are not included in energy expenditures. We used a fixed value of 1000 bits for data packet size $k$.

Figure 2 and Figure 3 show the computed routing paths for MST and LMST based routing schemes. One important point in the figures is that the nodes closer to the base station tend to send directly to the sink instead of choosing a closer neighbor. This is mainly because we include the receive cost in the link cost. As given in (4) there is no receive cost for the base station. If a node closer to the sink transmits to its closest neighbor, the transmitted node should also spend the energy required to receive one more packet. So if the MST's are computed with $(3,4)$, the nodes for which it is advantageous to send directly to the sink will choose the sink as their parent. As seen in the figures, our proposed algorithm approximates the original MST quite well. This is as expected since as stated in [10] the LMST structure has only $5 \%$ more edges compared to MST.

Figure 4 and Figure 5 show the timings of all node failures until the network is partitioned for networks of sizes 


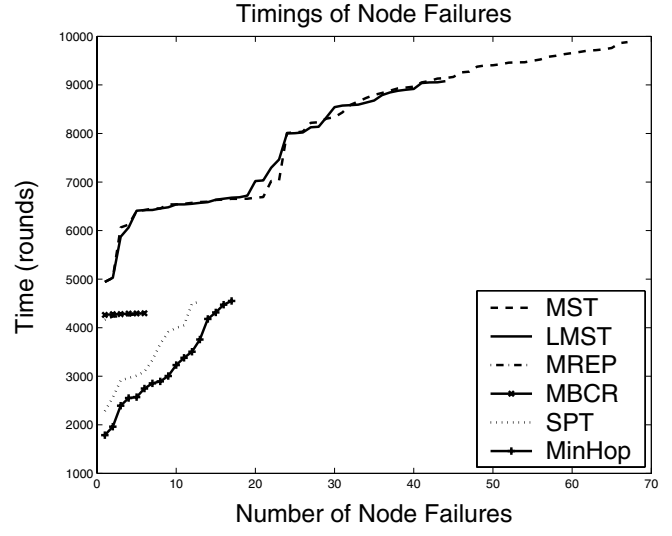

Figure 4. Timings of node failures-100 nodes

100 nodes and 50 nodes respectively. As depicted in the figures, MST gives a very good lifetime in terms of both first node failure and network partition time. Our LMST based approach gives almost the same results with MST based routing whereas in dense networks MST performs quite better. The power aware methods (MREP and MBCR) give better lifetime for first node compared with other shortest path based methods whereas minimum hop and minimum energy routing provides a connected network for a longer time compared to power aware methods.

Table 1 and Table 2 summarize the results for two different networks of sizes 50 and 100 respectively. In the tables AVG stands for the average node lifetime, FNF is the first node failure time, NPT shows the network partition time, and CNT stands for number of failed nodes when the network is partitioned. The simulation results show that our LMST based routing scheme improves the lifetime of the first node by $100 \%$ when compared with non-power aware shortest-path based algorithms, and provides significantly better lifetime for the first node when compared with poweraware algorithms.

The simulations also show that the LMST based approach improves the network partition time drastically. It can also be seen that the improvement of the LMST structure on the lifetime of the system is considerably large when

Table 1. Statistics for network of size 100.

\begin{tabular}{|c|c|c|c|c|}
\hline & AVG & FNF & NPT & CNT \\
\hline MST & 8191 & 4942 & 9881 & 67 \\
LMST & 7497 & 4942 & 9077 & 44 \\
MREP & 4240 & 4163 & 4282 & 6 \\
MBCR & 4283 & 4262 & 4297 & 6 \\
SPT & 3447 & 2283 & 4532 & 13 \\
MinHop & 3185 & 1787 & 4550 & 17 \\
\hline
\end{tabular}

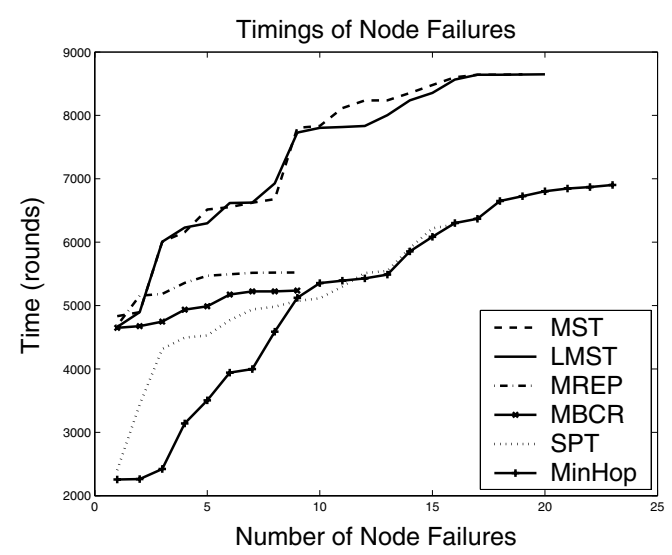

Figure 5. Timings of node failures -50 nodes

the number of nodes in the same area is increased. We can conclude that LMST approach is more effective in environments where the nodes are densely deployed.

\section{Conclusion}

In this paper we presented a new energy efficient routing algorithm that combines the desired properties of minimum spanning tree and shortest path tree based routing schemes. The proposed algorithm uses the advantages of the powerful LMST structure and provides simple solutions to the known problems in route setup and maintenance because of its distributed nature. The proposed algorithm is robust, scalable and self organizing. The algorithm is appropriate for systems where all the nodes are not in direct communication range of each other. We show through simulations that our algorithm outperforms some alternatives.

In our future work we will consider various improvements to our protocol. They will be all oriented toward reducing message overhead, without sacrificing performance significantly at the data aggregation time. Currently each route discovery packet has three fields. One of them contains the list of LMST neighbors. This can be avoided if RNG structure is used instead (an edge is in RNG if and only if it does not have the largest weight in any trian-

Table 2. Statistics for network of size 50.

\begin{tabular}{|c|c|c|c|c|}
\hline & AVG & FNF & NPT & CNT \\
\hline MST & 7361 & 4832 & 8648 & 19 \\
LMST & 7359 & 4662 & 8648 & 20 \\
MREP & 5323 & 4685 & 5522 & 9 \\
MBCR & 4983 & 4648 & 5236 & 9 \\
SPT & 4926 & 2403 & 6278 & 16 \\
MinHop & 5144 & 2256 & 6903 & 23 \\
\hline
\end{tabular}


gle). HELLO messages suffice for nodes to learn their RNG neighbors.

The distance field in the route discovery packet is related to the maintenance of the shortest weighted path from each sensor to the sink. This can be eliminated if each sensor node is simply linked to the very first parent node (from LMST or RNG structure), that is, to the first arriving route discovery message from the sink. Alternatively, the closest of candidate parent nodes may be selected, but in this case additional details must be provided to avoid loops and partitions and preserving tree structure. Even the later case may be beneficial (despite the need to preserve a field for, say, counting hop distance in path toward sink) when considering addition and deletion of sensor nodes from the network. Current implementation requires partial flooding through the network to maintain shortest weighted path property toward sink for all the network nodes. This may cause chain effect, the change in shortest weighted path in sensors which are far away from the area where insertion or deletion occurred. Our alternative proposal is to restrict maintenance hopefully only to 1-hop or possibly 2-hop neighbors of failed or added sensor. Parent of failed node may initiate route discovery only in order for children of failed node to receive new parent. If failed node was a 'bridge' between two network partitions then a recovery routing tasks may be issued to link these children again. This may be achieved by a routing procedure that guarantees delivery, such as GFG from [2]. In case of node additions, the task is much easier. If none of existing aggregation tree links was deleted from the LMST or RNG then the new node may only select the closest LMST or RNG neighbor as its parent, and be added as new leaf node. Otherwise new node becomes parent or child for all nodes affected (whose links from the current tree were broken). New node may become child of one such neighbor that preserved its parent, and become parent for the remaining affected neighbors. Details will be given in the journal version of this article.

\section{References}

[1] M. Bhardwaj, A. Chandrakasan, and T. Garnett. Upper bounds on the lifetime of sensor networks. In IEEE International Conference on Communications, pages 785-790, 2001.

[2] P. Bose, P. Morin, I. Stojmenović, and J. Urrutia. Routing with guaranteed delivery in ad hoc wireless networks. Wire- less Networks, 7(6):609-616, 2001.

[3] J. Chang and L. Tassiulas. Maximum lifetime routing in wireless sensor networks. In Proceedings of Advanced Telecommunications and Information Distribution Research Program, College Park, MD, 2000.

[4] J.-H. Chang and L. Tassiulas. Energy conserving routing in wireless ad-hoc networks. In IEEE INFOCOM 2000, pages 22-31, March 2000.

[5] W. R. Heinzelman, A. Chandrakasan, and H. Balakrishnan. Energy-efficient communication protocol for wireless microsensor networks. In 33rd Annual Hawaii International Conference on System Sciences, pages 3005 - 3014, 2000.

[6] C. Intanagonwiwat, R. Govindan, and D. Estrin. Directed diffusion: a scalable and robust communication paradigm for sensor networks. In Mobile Computing and Networking, pages 56-67, 2000.

[7] K. Kalpakis, K. Dasgupta, and P. Namjoshi. Maximum lifetime data gathering and aggregation in wireless sensor networks. In Proceedings of the 2002 IEEE International Conference on Networking (ICN'02), pages 685-696, August 2002.

[8] N. Li, J. C. Hou, and L. Sha. Design and analysis of an mst-based topology control algorithm. In INFOCOM, 2003.

[9] S. Lindsey and C. S. Raghavendra. Pegasis: Power-efficient gathering in sensor information systems. In IEEE Aerospace Conference, March 2002.

[10] F. J. Ovalle-Martinez, I. Stojmenovic, F. Garcia-Nocetti, and J. Solano-Gonzalez. Finding minimum transmission radii for preserving connectivity and constructing minimal spanning trees in ad hoc and sensor networks. J. Parallel Distrib. Comput., 65(2):132-141, 2005.

[11] C. E. Perkins and E. M. Royer. Ad-hoc on-demand distance vector routing. Proceedings of the 2nd IEEE Workshop on Mobile Computing Systems and Applications, page 90, 1999.

[12] V. Rodoplu and T. Meng. Minimum energy mobile wireless networks. IEEE J. Select. Areas Commun., 17(8):1333 1344, Aug. 1999.

[13] S. Singh, M. Woo, and C. S. Raghavendra. Power-aware routing in mobile ad hoc networks. In Mobile Computing and Networking, pages 181-190, 1998.

[14] I. Stojmenovic and X. Lin. Power-aware localized routing in wireless networks. IEEE Transactions on Parallel and Distributed Systems, 12(11):1122-1133, 2001.

[15] H. Ö. Tan and I. Korpeoglu. Power efficient data gathering and aggregation in wireless sensor networks. SIGMOD Record, 32(4):66-71, 2003.

[16] C.-K. Toh. Maximum battery life routing to support ubiquitous mobile computing in wireless ad hoc networks. IEEE Communications Magazine, June 2001. 